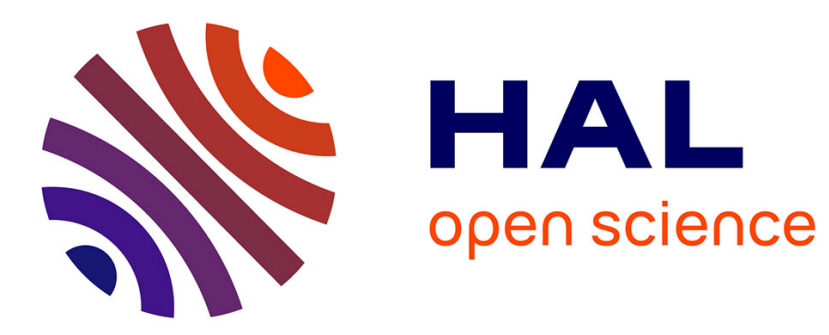

\title{
Full-parallel architecture for turbo decoding of product codes
}

\author{
Christophe Jego, Patrick Adde, Camille Leroux
}

\section{To cite this version:}

Christophe Jego, Patrick Adde, Camille Leroux. Full-parallel architecture for turbo decoding of product codes. Electronics Letters, 2006, 42 (18), pp.1052 -1053. 10.1049/el:20062168 . hal-00538604

\section{HAL Id: hal-00538604 \\ https://hal.science/hal-00538604}

Submitted on 23 Nov 2010

HAL is a multi-disciplinary open access archive for the deposit and dissemination of scientific research documents, whether they are published or not. The documents may come from teaching and research institutions in France or abroad, or from public or private research centers.
L'archive ouverte pluridisciplinaire HAL, est destinée au dépôt et à la diffusion de documents scientifiques de niveau recherche, publiés ou non, émanant des établissements d'enseignement et de recherche français ou étrangers, des laboratoires publics ou privés. 


\title{
Full-parallel architecture for turbo decoding of product codes
}

\author{
C. Jégo, P. Adde and C. Leroux
}

A full-parallel architecture for turbo decoding, which achieves ultra high data rates when using product codes as error correcting codes, is proposed. This architecture is able to decode product codes using binary $\mathrm{BCH}$ or m-ary Reed Solomon component codes. The major advantage of our architecture is that it enables the memory blocks between all half-iterations to be removed. Moreover, the latency of the turbo decoder is strongly reduced. In fact, the proposed architecture opens the way to numerous applications such as optical transmission and data storage. In particular, our block turbo decoding architecture can support optical transmission at data rates above $10 \mathrm{~Gb} / \mathrm{s}$.

Introduction: In recent years, turbo codes [1] have been adopted by several digital communication applications. In fact, they are particularly attractive to increase transmission rates and/or to guarantee the Quality of Service (QoS). Currently, research is under way to use turbo codes to protect data stored on hard drive or DVD and in fiber optical transmission. The earliest FEC for optical communication [2] employed the well-known Reed-Solomon (RS) codes to recover the degradation in bit error rate (BER) due to the effects of fiber nonlinearity and polarization-dependent phenomena. A net coding gain of around $6 \mathrm{~dB}$ is provided by the RS $(255,239)$ code. Very high-speed data transmission developed for fiber optical networking systems necessitate the 
implementation of ultra high-speed FEC architectures to meet the continuing demands for ever higher data rates. Currently, the $\mathrm{RS}(255,239)$ code can be used in ultra high-speed (40 Gb/s [3] and $80 \mathrm{~Gb} / \mathrm{s}$ [4]) fiber optic systems. More powerful FECs as Block Turbo Codes (BTC) have a theoretical potential net coding gain of around $10 \mathrm{~dB}$ with a redundant overhead of less than $25 \%$ [5]. Typically, realistic block turbo codes can operate at less than $1 \mathrm{~dB}$ from the Shannon limit for a binary symmetric channel. In 2005, Mitsubishi Electric announced the development of the first block turbo decoder for $10 \mathrm{~Gb} / \mathrm{s}$ optical transmission [6].

Previous work: Many block turbo decoder architectures have been previously designed. The classical approach involves decoding all the rows or all the columns of a matrix before the next half-iteration. When an application requires high-speed decoders, an architectural solution is to cascade SISO elementary decoders for each half-iteration. In this case, memory blocks are necessary between each half-iteration to store channel data and extrinsic information. Each memory block is composed of four memories of $q n^{2}$ symbols where $q$ is the number of bits to quantify the matrix symbols. Thus, duplicating a SISO elementary decoder $\left(e_{-} d e c\right)$ results in duplicating the memory block which is very costly in terms of area. In 2002, a new architecture for turbo decoding product codes was proposed [7]. The idea is to store several data at the same address and to perform parallel decoding to increase the data rate. However, it is necessary to process these data by row and by column. Let us consider $m$ adjacent rows and $m$ adjacent columns of the initial matrix. The $m^{2}$ data constitute a word of the new matrix that has $m^{2}$ 
times fewer addresses. This data organization does not require any particular memory architecture. The results obtained show that the turbo decoding is increased by $m^{2}$ when $m$ elementary decoders processing $m$ data simultaneously are used and its latency is divided by $m$. The area of the $m$ elementary decoders $\left(m-e_{-} d e c\right)$ is increased by $m^{2} / 2$ while the memory is constant.

Full-parallel turbo decoding principle: The codewords of all rows (or all columns) of a matrix can be decoded in parallel. If the architecture is composed of $n$ elementary decoders, an appropriate treatment of the matrix enables the elimination of the reconstruction of the matrix between each decoding. Let $i$ and $j$ be the indices of a row and a column of the $n^{2}$ matrix. In full-parallel processing, the row decoder $i$ begins the codeword decoding by the symbol in the $i^{\text {th }}$ position. Moreover, each row decoder processes the codeword symbols by increasing the index by one modulo $n$. Similarly, the column decoder $j$ begins the codeword decoding by the symbol in the $j^{\text {th }}$ position. Besides, each column decoder processes the codeword symbols by decreasing the index by one modulo $n$. Thus only one time cycle is necessary between two successive decoding the matrix. The full-parallel decoding of a $n^{2}$ product code matrix is detailed in Figure 1. A similar strategy was previously presented in [8]. In this case, the conflicts of $n$ independent RAM memories are eliminated by the appropriate treatment of the matrix. The elementary decoder latency can be defined as the symbol number processed by the decoder during the decoding of one symbol. This latency $L$ depends on the structure of the elementary decoder and the $n$ codeword length. As the 
reconstruction matrix is removed, the latency between row and column decoding is null.

Full-parallel turbo decoder for product codes: The major advantage of our full-parallel architecture is that it enables the memory block of $4 q n^{2}$ symbols between each half-iteration to be removed. But, the codeword symbols exchanged between the row and column decoders have to be switched. One solution is to use a connection network for this task. In our case, we have chosen an Omega network. The Omega network is one of several connection networks that are used in parallel machines [9]. It is composed of $\log _{2} n$ stages, each having $n / 2$ exchange elements. In fact, the Omega network complexity in terms of number of connections and of $2 * 2$ switch transfer blocks is $n^{*} \log _{2} n$ and $\left(n^{*} \log _{2} n\right) / 2$ respectively. For example, the equivalent gate complexity of a $32 \times 32$ network can be estimated to be 200 per exchange bit. The proposed full-parallel architecture for product codes is presented in Figure 2. It is composed of cascaded modules for the block turbo decoder. Each module is dedicated to one iteration. However, it is possible to process several iterations by a same module. In our approach, $2 n$ elementary decoders and 2 connection networks are necessary for one module. In fact, the full-parallel turbo decoder complexity essentially depends on the complexity of the elementary decoder. In order to compare our architectural solution with the previous solutions, Table 1 gives the features of these architectures. The features depend on different parameters: symbol codeword $n$, decoding iteration $i t$, elementary decoder throughput $D_{\text {ref, elementary }}$ decoder latency $L$, symbol quantization bits $q$ and adjacent symbol group $m$. 
The e_dec and $m$-e_dec architecture types correspond to the classical solution and the solution in [7] respectively.

Towards the implementation of architectures for ultra high rates: By using the full-parallel decoding principle, block turbo decoders using $\mathrm{BCH}$ component codes have been implemented. An architecture of $\mathrm{BCH}(32,26)^{2}$ product codes with single correction power was synthesized. The decoding algorithm is chosen with $q=4$ quantization levels, 8 test vectors, 1 competitor and $i t=4$ iterations. The elementary decoding of a codeword is split into three pipelined phases. Each phase requires $32 / \mathrm{m}$ clock periods and the elementary decoder latency is equal to $64 / \mathrm{m}$ clock periods. Syntheses were performed using the Synopsys tool with an STMicrolectronics $0.09-v m$ CMOS process target library. Two architecture types were chosen: e_dec as the reference and 4e_dec where $m=4$ symbols are simultaneously processed by an elementary decoder. Elementary decoders have a clock period equal to 2 ns which corresponds to a frequency of $500 \mathrm{MHz}$. The estimated area complexity in terms of equivalent gates for the two elementary decoders are: 4400 for $\mathrm{BCH}(32,26)$ e_dec and 5700 for $\mathrm{BCH}(32,26)$ 4-e_dec. This complexity includes all the elementary decoder elements (processing and memorization). The processing unit gate numbers of the block turbo decoders are equivalent between previous and proposed architectures: 1.13 and 1.45 millions for e_dec and 4-e_dec respectively. The latency is strongly reduced for the proposed architecture. It decreases from 270336 to 512 for $e_{-}$dec and from 5120 to 128 for $4-e \_d e c$. The memory complexity of the previous architecture in terms of equivalent gates is 126400 . It corresponds to 10 percent of 
$\mathrm{BCH}(32,26)^{2}$ block turbo decoder complexity. On the other hand, the equivalent gate complexity of connection networks is only 5600 for the proposed architecture of $\mathrm{BCH}(32,26)^{2}$ block turbo decoder.

Conclusion: A full-parallel turbo decoding architecture for product codes has been proposed. This architecture enables the memory blocks between all half-iterations to be removed. Moreover, the latency of the turbo decoder is strongly reduced. The ultra high-speed FEC architectures obtained meet the demands for ever-higher data rates. In particular, our architectural solution can support optical transmission at data rates above $10 \mathrm{~Gb} / \mathrm{s}$. In this context, using more powerful FEC as block turbo codes open up new opportunities for the next generation of optical communication systems.

\section{References}

1 Berrou C., Glavieux A., Thitimajshima P.: 'Near Shannon limit error correcting coding and decoding: Turbo Codes', IEEE International Conference on Communication ICC93, vol. 2/3, May 1993.

2 Azadet K., Haratsch E.F., Kim H., Saibi F., Saunders J.H., Shaffer M.S., Song L., Meng-Lin Y.: 'Equalization and FEC techniques for optical transceivers', Solid-State Circuits, IEEE Journal of Volume 37, Issue 3, March 2002, pp. 317-327.

3 Leilei S., Meng-Lin Y., Shaffer M.S.: '10- and 40-Gb/s forward error correction devices for optical communications', Solid-State Circuits, IEEE Journal of Volume 37, Issue 11, Nov. 2002, pp. 1565-1573.

4 Hanho L.: 'A high-speed low-complexity Reed-Solomon decoder for optical communications', Circuits and Systems II: Express Briefs, IEEE Transactions on Volume 52, Issue 8, Aug. 2005, pp. 461-465.

5 Mizuochi T.: 'Recent Progress in Forward Error Correction for Optical Communication Systems', IEICE Transactions on Communications, Volume E88-B, Number 5, May 2005.

6 Tagami H., Kobayashi T., Miyata Y., Ouchi K., Sawada K., Kubo K., Kuno K., Yoshida H., Shimizu K., Mizuochi T., Motoshima K., 'A 3-bit soft- 
decision IC for powerful forward error correction in 10-Gb/s optical communication systems', Solid-State Circuits, IEEE Journal_of Volume 40, Issue 8, Aug. 2005, pp. 1695-1705.

7 Cuevas J., Adde P., Kerouedan S., Pyndiah R.: 'New architecture for high data rate turbo decoding of product codes', GLOBECOM 2002, November 2002, pp. 139-143.

8 Zhipei Chi; Parhi, K.K.: 'High speed VLSI architecture design for block turbo decoder', ISCAS 2002, IEEE International Symposium on Volume 1, May 2002, pp. 901-904.

9 Lawrie D. H.: 'Access and alignment of data in an array processor', IEEE Trans. Computer, vol. C-24, no. IO, December 1975, pp. 1145-1155.

\section{Authors' affiliations:}

C. Jégo, P. Adde and C. Leroux (GET/ENST Bretagne, CNRS TAMCIC UMR 2872, Brest, France)

\section{Figure captions:}

Fig. 1 Full-parallel decoding of a product code matrix

Fig. 2 Full-parallel architecture for product codes

Table 1 : Features of different architectures for block turbo decoding 
Figure 1

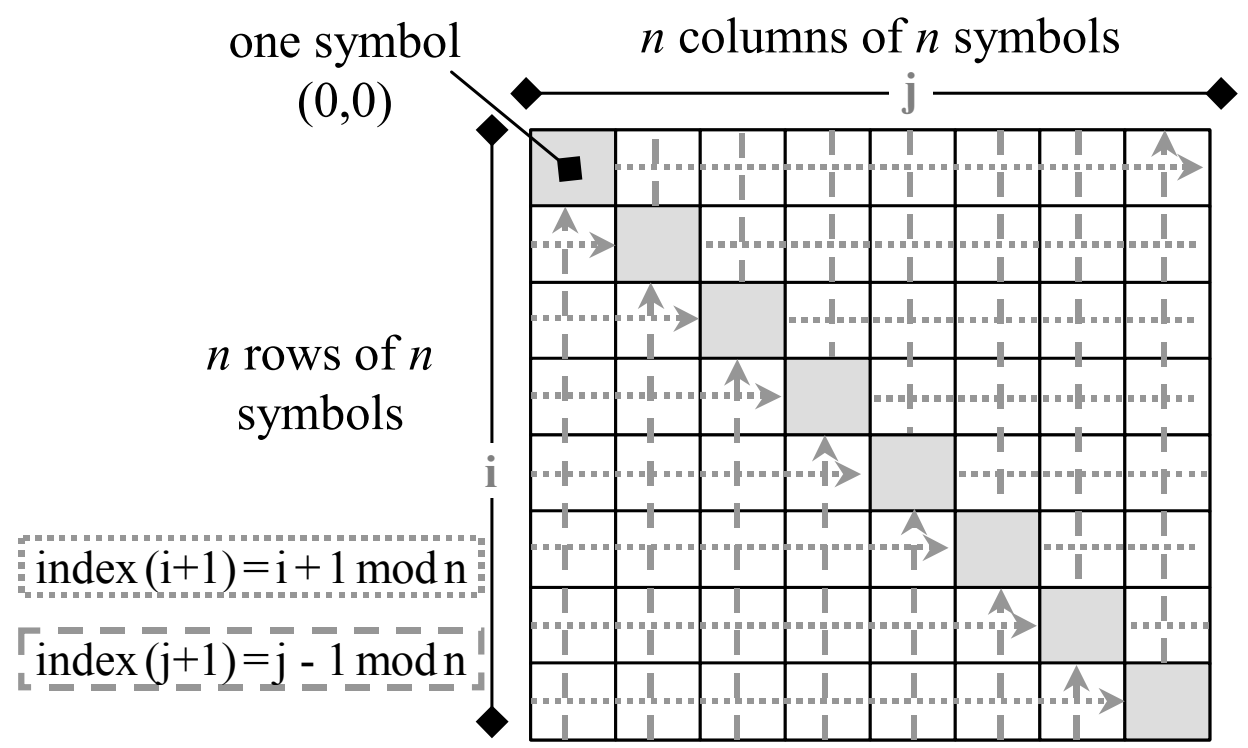

Figure 2

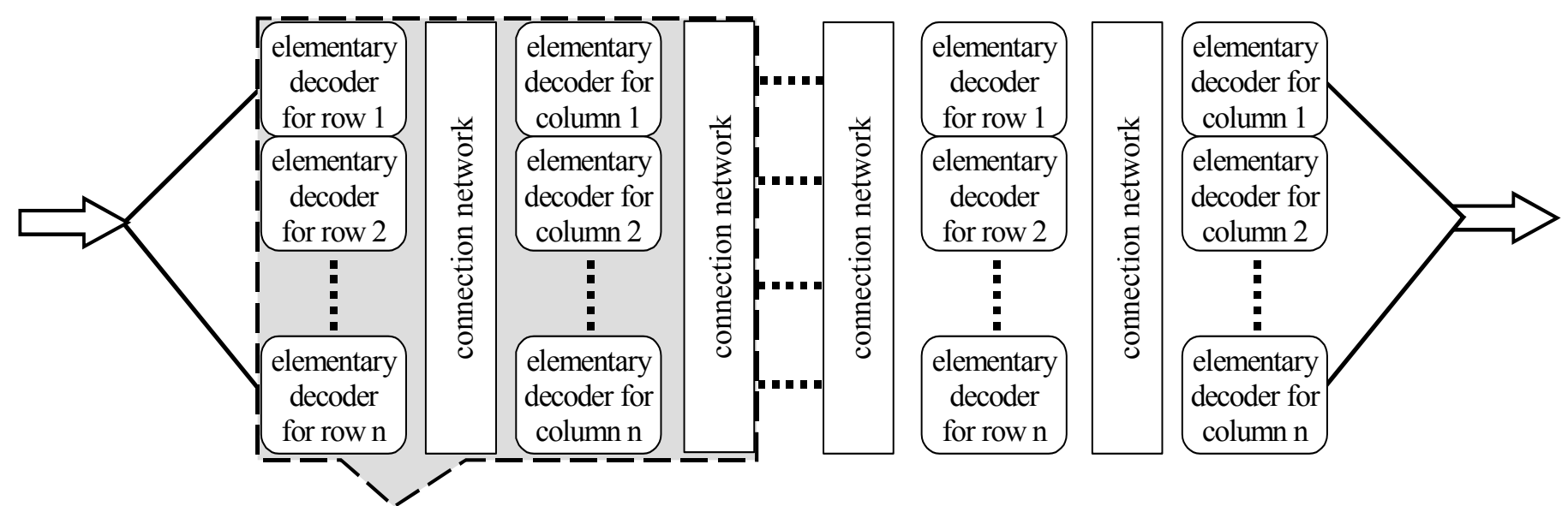

a module for one iteration 
Table 1

\begin{tabular}{|c|c|c|c|c|}
\hline & \multicolumn{2}{|c|}{ Previous architectures } & \multicolumn{2}{|c|}{ Proposed architectures } \\
\hline & e_dec & m-e_dec & e_dec & m-e_dec \\
\hline $\begin{array}{c}\text { latency } \\
\text { (symbol number) }\end{array}$ & $\begin{array}{c}n *\left(2 i t * n^{2}+\right. \\
2 i t * L)\end{array}$ & $\begin{array}{c}n / m *\left\{2 i t *\left(n^{2} / m^{2}\right)\right. \\
+2 i t *(L / m)\}\end{array}$ & $2 i t * L$ & $2 i t *(L / m)$ \\
\hline $\begin{array}{c}\text { throughput } \\
(\mathrm{Gb} / \mathrm{s})\end{array}$ & $n *\left(2 i t * D_{r e f}\right)$ & $n / m *\left(m^{2} * 2 i t * D_{r e f}\right)$ & $n *\left(2 i t * D_{\text {ref }}\right)$ & $n / m *\left(m^{2} * 2 i t * D_{r e f}\right)$ \\
\hline e_dec number & $n * 2 i t$ & $n / m *(m * 2 i t)$ & $n * 2 i t$ & $n / m *(m * 2 i t)$ \\
\hline $\begin{array}{c}\text { memory size } \\
(\mathrm{Kb})\end{array}$ & $2 i t * 4 q n^{2}$ & $2 i t * 4 q n^{2}$ & 0 & 0 \\
\hline \begin{tabular}{|c|} 
connection \\
network number
\end{tabular} & 0 & 0 & $2 i t-1$ & $2 i t-1$ \\
\hline
\end{tabular}

